\title{
Perspectives for Improvement of the Thymic Microenvironment through Manipulation of Thymic Epithelial Cells: A Mini-Review
}

\author{
Ailin Lepletier $^{\mathrm{a}, \mathrm{b}}$ Ann P. Chidgey ${ }^{\mathrm{b}}$ Wilson Savino $^{\mathrm{a}}$

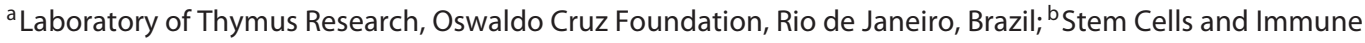 \\ Regeneration Laboratory, Department of Anatomy and Developmental Biology, Monash University, \\ Melbourne, Vic., Australia
}

\section{Key Words}

Aging $\cdot$ Thymic involution · Immune senescence $\cdot$ Thymic epithelial cells · Intrathymic circuitry · FoxN1 expression · Thymic epithelial progenitor cells

\begin{abstract}
Thymic involution during aging is a major reason for the decreased production of naive $T$ cells and reduced immunity. Alterations within the thymic microenvironment, characterized by the loss of function of thymic epithelial cells (TECs) and fibro-adipogenetic transformation, seem to underlie this process, mainly through declining communication between thymic stromal cells and developing thymocytes. Specifically, the signaling mediated by cytokines and hormones secreted by TECs declines during aging. Many therapies based on the manipulation of growth factors and hormones have succeeded in partially recovering the lymphoid compartment and promoting thymic function. However, considering that aging-induced thymic involution is multifactorial, the thymic reestablishment achieved with treatments that target isolated pathways is incomplete and transitory. Here, we discuss the development of three novel approaches for potentially sustained thymic recovery: the induction of sustained forkhead box N1 expression, the activation of endogenous thymic epithelial progenitor cells (TEPCs), and the generation of TEPCs from pluripotent stem
\end{abstract}

cells. Combined approaches targeting both TECs and lymphoid cells will provide a potentially more effective strategy for sustained rejuvenation of the thymus.

(C) 2015 S. Karger AG, Basel

\section{Introduction}

The thymus is one of the first organs to degenerate postnatally, with thymic involution beginning early in life and significantly impacting on immune system aging [1]. It has been reported in all jawed vertebrates, which indicates that this is an evolutionary ancient and conserved event. Aging-induced thymus degeneration is associated with a reduction in the cellularity of thymic epithelial cells (TECs) and thymocyte subsets, fibro-adipogenetic transformation, loss of tissue structure, and abnormal architecture, resulting in a decline of naive T-cell export. The consequent changes in the peripheral T-cell compartment are believed to be, at least in part, responsible for the clinical signs of immunosenescence. Accordingly, elderly individuals show a decreased immunoresponsiveness, are more susceptible to new threats, and are less able to control infection [2]. Furthermore, immune recovery following cytoablative treatments can be severely delayed in middleaged and elderly patients, leading to increased morbidity and mortality due to opportunistic infections [3].

\section{KARGER 125}

(c) 2015 S. Karger AG, Base

0304-324X/15/0616-0504\$39.50/0

E-Mail karger@karger.com

www.karger.com/ger
Wilson Savino

Laboratory of Thymus Research, Oswaldo Cruz Foundation

Ave. Brasil 4365

Manguinhos, Rio de Janeiro, RJ 21045-900 (Brazil)

E-Mail savino@ fiocruz.br 
Table 1. Hallmarks of thymus atrophy during aging of humans and mice

\begin{tabular}{|c|c|}
\hline Humans & Mice \\
\hline Progressive decline of recent thymic emigrants [25] & Decreased thymic output [36] \\
\hline $\begin{array}{l}\text { Enlarged PVS due to replacement of lymphoid cells } \\
\text { by adipocytes [71] }\end{array}$ & $\begin{array}{l}\text { Increased number of adipocytes in thymic } \\
\text { parenchyma expressing CCR } 5 \text { and its ligands [72] }\end{array}$ \\
\hline $\begin{array}{l}\text { Increase in LIF, stem cell factor, M-CSF, and } \\
\text { oncostatin M by TEC and adipocytes [29] }\end{array}$ & Decreased expression of Wnt4 as FoxN1 in TEC [58] \\
\hline $\begin{array}{l}\text { Decrease in IL-2, IL-9, IL-10, IL-13, and IL-14 } \\
\text { production by TEC [29] }\end{array}$ & Reduction of cTEC and mTEC MHCII ${ }^{\text {hi }}[12]$ \\
\hline \multirow[t]{2}{*}{$\begin{array}{l}\text { Increase in angiogenetic and endothelial factors by } \\
\text { adipocytes [73] }\end{array}$} & $\begin{array}{l}\text { Reduced proliferative TEC staining with Ki67 and } \\
\text { increased level of apoptotic TECs [23] }\end{array}$ \\
\hline & $\begin{array}{l}\text { Differentiation of fibroblast into preadipocyte } \\
\text { through the increase of PPAR } \gamma[17]\end{array}$ \\
\hline
\end{tabular}

The precise mechanisms that underlie age-dependent thymus atrophy are still unclear. The thymus relies on the supply of lymphoid precursors from the bone marrow (BM) for T-cell development. Therefore, intrinsic age-related alterations in the hematopoietic compartment, such as a reduced lymphoid-to-myeloid lineage ratio with age, likely play a role in exacerbating thymic involution [4]. However, it appears that the thymic microenvironment itself is also responsible for influencing many of the defects exhibited by the T-cell compartment $[5,6]$.

Accordingly, studies on young adults, thymectomized during early childhood, demonstrate that despite their young age, some patients exhibit profound long-lasting perturbations, typically associated with advanced aging (i.e. $>75$ years old), as decreased $\mathrm{T}$-cell count and very low naive $\mathrm{T}$-cell frequency, reduced $\mathrm{T}$-cell repertoire diversity, and increased numbers of highly differentiated memory $\mathrm{T}$ cells with shortened telomeres $[7,8]$. In contrast to these studies, van Gent et al. [9] showed a normalization of the T-cell pool after 5 years of surgery. A slow recovery of the thymic tissue, detected by magnetic resonance imaging scans, was followed by the reestablishment of naive $\mathrm{CD} 4^{+}$and $\mathrm{CD} 8^{+} \mathrm{T}$-cell counts and TRECs to normal ranges. However, the findings of this study were not uniformly confirmed.

Recent approaches to rejuvenate the immune system used in preclinical assays and in some clinical trials include modulation of the endocrine system such as sex steroid ablation or administration of growth factors such as growth hormone $(\mathrm{GH})$, interleukin 7 (IL-7) and IL-22 [for a review, see 10]. Whilst these approaches impact on improving thymopoiesis for the duration of treatment in preclinical models, it is unclear whether any parallel transient improvements in the TEC compartment are due to direct or secondary effects. Therefore, targeted interventions to rejuvenate the thymic epithelial compartment itself have recently emerged as a new generation of approaches.

\section{Hallmarks of Thymic Involution: A Perspective from TEC-Related Mechanisms}

Thymic senescence precedes that of other organs, resulting in an altered development and exportation of naive $\mathrm{T}$ cells to the periphery [11]. The mechanisms that underlie TEC-mediated transformations leading to the progressive decline in thymic function during aging are reviewed below, and findings in humans and mice are separately summarized in table 1 .

\section{Disruption of the Epithelial Tridimensional Network}

Previous studies have demonstrated a clear loss in thymic epithelial tissue from as early as the first year of life, with $>75 \%$ of the decline occurring up to middle age, followed by a gradual further decline [1]. TECs in aging mice have lower proliferative capacity and a higher rate of apoptosis compared with the values recorded in young animals [12]. As a consequence, there is a progressive increase in perivascular nonepithelial spaces, an associated breakdown of the defined cortical medullary junction, and a shift in the medullary-versus-cortical TEC ratio towards the cortical subset (cTEC) [12]. 
Fig. 1. Mechanisms underlying thymus atrophy during aging. The cross-talk between TECs and thymocytes is essential for thymus homeostasis. During aging, this communication is disrupted and the result is cellular depletion in both lymphoid (decrease in numbers of all thymocyte subsets) and epithelial compartments. Due to a depletion in $\mathrm{TEC}^{\text {hi }}$, the ratio of $\mathrm{TEC}^{\text {hi }}$ / $\mathrm{TEC}^{\text {int/lo }}$ gradually decreases with age. The impairment of TEC subsets seems to be at least partially related to their conversion to adipocytes, a phenomenon that seems to occur mainly in the thymic medulla.

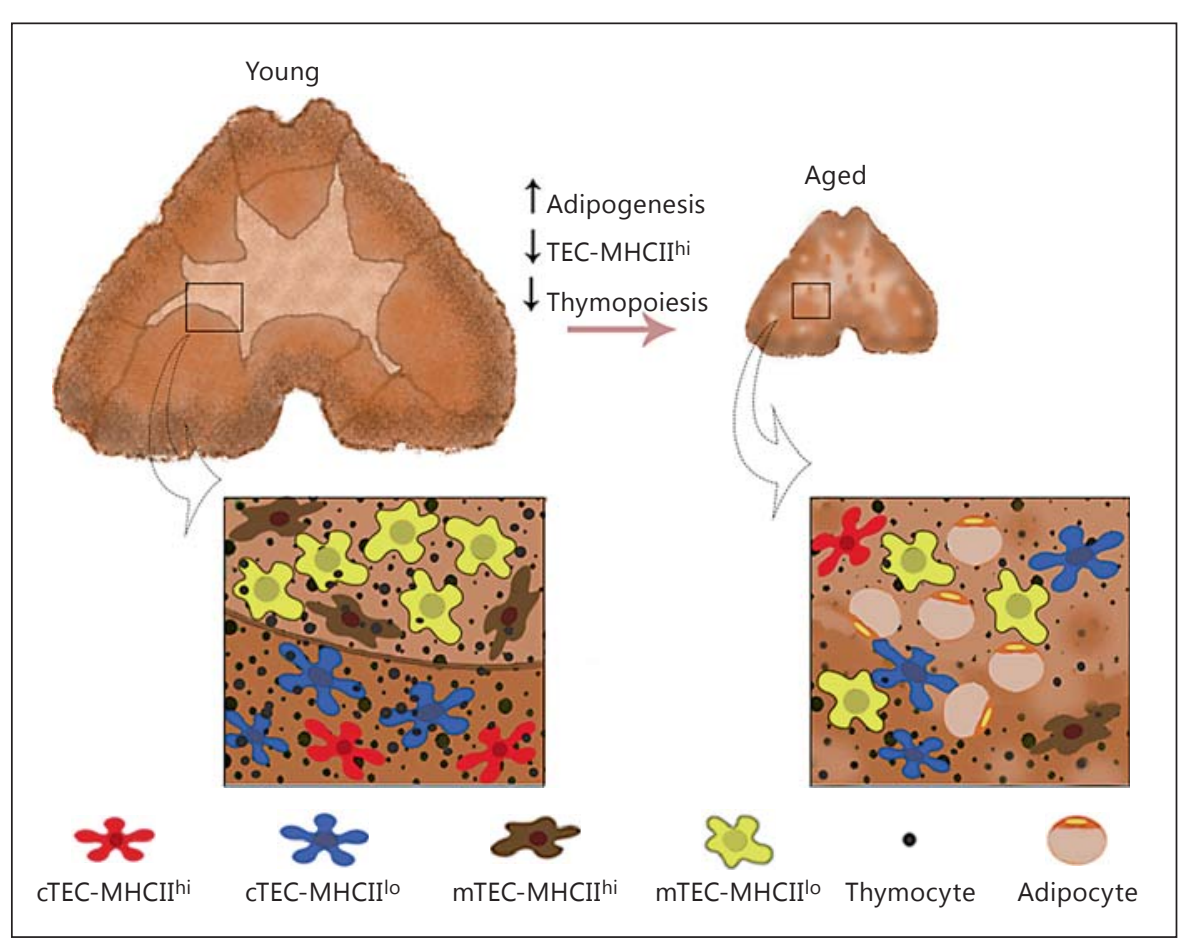

An impairment in the cross-talk between TEC and developing thymocytes seems to be a detrimental contributing factor for epithelial structural disruption during aging. Several key inductive interactions between these cell types have been reported. The ratio of TECs with a high surface expression of major histocompatibility complex class II (MHCII;MHCII ${ }^{\text {hi }}$ ) to TECs with an intermediate/ low MHCII cell surface expression $\left(\mathrm{MHCII}^{\mathrm{int} / \mathrm{lo}}\right)$ gradually diminishes with age [12], as illustrated in figure 1. Thymocyte interaction is required for the upregulation of MHCII expression during ontogeny; therefore, this change may reflect the reduced levels of thymopoiesis. Medullary TEC (mTEC) formation, differentiation, and expansion require signaling from thymocytes through receptor activator of nuclear factor kappa-B ligand and CD40L [13].

The decreased availability of thymic epithelial niches for thymocyte progenitors in the involuted thymus or the deterioration in quality of these niches may account in part for reduced thymopoiesis in aging. Evidence supporting this notion comes from studies demonstrating that an increased TEC number produces a proportional increase in thymus size. The expression of cyclin D1 under a keratin 5 gene promoter in TEC results in a dramatic increase in thymus size and is sufficient to delay or prevent involution [14]. Using an embryo fusion chime- ra-based approach, Jenkinson et al. [15] demonstrated that aging-associated thymus atrophy is related both to the restriction of thymus growth due to a reduction in the provision of growth factors as well as to a decrease in the proliferation of thymic epithelial progenitor cells (TEPCs). It seems that niche availability serves as a key determinant for thymus output and that restriction in the number and/or function of thymic microenvironmental niches is a critical cellular and structural component of thymic involution.

As thymic involution proceeds age-induced alterations in the BM, intrinsic defects in BM-derived hematopoietic stem cells (HSCs) and their capacity to generate high-quality lymphoid progenitors are likely to be contributing rather than initiating factors in thymic involution. Transferring young HSCs or early T-lineage progenitors (ETPs) from young mice into aged recipients alone does not restore thymic compartmental structure [16].

\section{Process of Fibro-Adipogenesis}

Thymic aging is associated with TEC decline and an increase in thymic fibroblast and adipocyte numbers. The adipocyte tissue accumulation is typically accompanied by an expansion of lipid-bearing cells within the thymic medulla [17] (fig. 1). Additionally, adipocyte infiltration 
is observed in several thymic zones, including the perivascular nonepithelial space, the interlobular septa, the capsular region, and the subcapsular cortex [11]. Inhibition of thymic adipogenesis by calorie restriction has been shown to reduce age-related thymic involution and increase thymic function [17].

Although the mechanisms involved in adipogenesis along with thymic involution are not completely understood, there are indications that adipocyte accumulation during aging results from TEC conversion. Recent studies employing genetic fate mapping suggest that a given TEC subset can transdifferentiate into fibroblasts and adipocytes during aging, via the activation of an intrathymic epithelial-mesenchymal transition (EMT) process [18]. Actually, certain EMT cells that appear to commit to the adipocyte lineage in the aging thymus express peroxisome proliferator-activated receptor gamma (PPAR $\gamma$ ) and unilocular lipid droplets and appear to commit to the adipocyte lineage [18]. The elevation of PPAR $\gamma$ and its upstream activator xanthine oxidoreductase (XOR) seems to drive thymus atrophy through the induction of ectopic adipogenesis. Accordingly, the specific inhibition of PPAR $\gamma$ in thymic stromal cells induced reduction in EMT, preventing their adipogenetic transformation in an XOR-dependent mechanism and leading to an increased thymic export [17].

These data led us to conceive that the loss of TEC phenotype and the emergence of TEC-derived fibro-adipogenetic precursors may have direct implications in compromising thymopoiesis during aging.

\section{Thymocyte Development Arrest}

In the adult thymus, the fully developed tridimensional (3D) TEC network provides a unique microenvironment for hematopoietic progenitor cells, allowing thymic colonization as well as intrathymic T-cell migration and differentiation. In this respect, this 3D disposition, which is essential for thymocyte development, seems to be related to TEC induction of Delta-like (Dl) 1 and Dl4 and the consequent activation of Notch signaling in ETPs [19]. These progenitors are the earliest intrathymic precursors contained within the $\mathrm{CD} 4 \mathrm{CD}^{-}(\mathrm{DN})$ compartment and are characterized as DN1a/b cells, expressing the markers $\mathrm{CD} 44^{+} \mathrm{CD} 25^{-} \mathrm{CD} 117^{+} \mathrm{CD} 24^{\text {int }}$. Aging mice have fewer ETP numbers, with reduced proliferation and differentiation potential. While ETP obtained from young mice are able to differentiate into all the stages of T-cell development when seeded into fetal thymic organ cultures [20], aging ETP show a reduction of T-cell differentiation activity, associated with an increased frequency of

Improvement of the Thymic

Microenvironment through TECs apoptosis and a reduced expression of $\mathrm{Ki}^{+} 7^{+}$[21]. These age-induced alterations in ETP seem to result in part from intrinsic mechanisms, with the increased expression of the Ink4a tumor suppressor gene [22].

It has also been speculated that the reduction in ETP population observed in the aging mouse thymus is associated with impairment in the recruitment of BM-derived hematopoietic progenitor cells, mediated mainly by the CCL25 chemokine, produced by TEC and the expression of P-selectin by specialized thymic venules. However, it has been shown that recruitment of lymphoid precursor cells (LPCs) from the bloodstream into the thymus is not impaired during aging and that the expression of $\mathrm{P}$-selectin and CCL25 is not reduced [23]. Also, LPCs from aging mice could normally seed the young thymus in a system of kidney capsule transplantation [23].

Besides ETPs, many studies have highlighted further age-related changes within the later stages of DN thymocyte development, with the observation of a decrease in the proportion of $\mathrm{DN} 2\left(\mathrm{CD} 44^{+} \mathrm{CD} 25\right)$ and $\mathrm{DN} 3$ $\left(\mathrm{CD} 44^{+} \mathrm{CD} 25^{+}\right)$cells [24]. DN fluctuation seems to directly impact the generation of double-positive thymocytes, the subsequent stage of thymocyte development. A worsening communication between the cortical and medullary thymic microenvironments with developing thymocytes during aging also results in the loss of selfversus non-self-recognition, due to failure of selection processes, and decreased or an inherent inability for Tcell receptor rearrangement [24].

With respect to mature thymocytes, although the thymic export of both CD4 and CD8 T cells has been reported to be altered during senescence, observations in humans and murine models have outlined a more dramatic decline in the CD8 subset [25], suggesting that aging-induced alterations in the thymic microenvironment differentially regulate the development of conventional T cells.

\section{Role of TEC-Mediated Cytokine and Hormonal Signaling during Thymic Senescence}

Many cytokines and hormones are secreted by the thymic microenvironment, providing constitutive processes such as migration and development of thymocytes, in addition to regulating cell numbers in the T-cell pools [26-28]. Age-dependent TEC transformations result in an altered secretory pattern that seems to underlie different aspects of thymic involution, as will be discussed below. 


\section{IL-6 Family Cytokines}

Cytokines of the IL- 6 proinflammatory family produced by TEC include IL-6, leukemia inhibitory factor and oncostatin M. Studies evaluating and profiling cytokines in human thymus during aging have demonstrated a shift in cytokine expression patterns to predominantly IL-6 family cytokines by thymic adipocytes and TECs [29]. The increased expression of leukemia inhibitory factor and IL- 6 further correlates with decreased production of recent thymic emigrants. Importantly, the administration of leukemia inhibitory factor, oncostatin M or IL-6 in mice results in thymic involution [29], suggesting a putative role of inflammation in thymus atrophy during aging.

\section{Transforming Growth Factor $\beta$}

Parallel to the cytokines of the IL- 6 family, the intrathymic expression of transforming growth factor $\beta$ (TGF- $\beta$ ) is also increased during aging [29]. TGF- $\beta$ signaling plays an age-dependent negative role in controlling thymic aging and cellularity, inhibiting the IL-1-, IL2- and IL-7-dependent thymocyte proliferation [30] as well as the maturation of the double-positive subset [31]. The physiological process of thymic senescence is attenuated in mice deficient for the expression of TGF- $\beta$ RII on TECs but not on thymocytes [32]. The lack of TGF- $\beta$ mediated signaling in TECs is correlated with an increase in the absolute number of thymocytes during puberty, and it also improves thymic reconstitution after irradiation [32]. These data reveal that, rather than directly affecting thymocytes, TGF- $\beta$ acts on the thymic microenvironment, modulating TEC capacity to support thymopoiesis. On the other hand, it has been shown that a block in canonical TGF- $\beta$ signaling by the loss of Smad4 expression in TECs leads to qualitative changes in TEC function and a progressively disorganized thymic microenvironment [33]. Although TGF- $\beta$ signaling exerts a main role in the induction of fibrosis in many organs [34], it is unclear if the fibrotic and fatty changes that culminate in TEC transformation into adipose tissue during aging are related to the local increase of this cytokine.

\section{Interleukin 7}

IL-7 signaling is required to induce T-cell receptor $\beta$ rearrangement in $\mathrm{DN}$ thymocytes and for the survival of these cells [35]. Accordingly, IL-7-deficient mice present a blockage at the DN1 to DN2 transition and subsequent loss of thymic output [36]. Since these changes are also observed in old mice, it is hypothesized that decreased IL-7 signaling in the thymus is involved in age-related thymic involution. In this respect, the intrathymic expression of IL-7 declines with aging in the thymic microenvironment [37], whereas neutralization of IL-7 in young mice induces reversible thymus atrophy [38]. The specific role of locally produced IL-7 in thymic involution during aging was assessed with the injection of IL7 -secreting stromal cells into the thymi of recipient aging mice [39]. Although the increased local concentration of IL-7 maintained the first step of thymopoiesis at a level far higher than was seen in age-matched controls, there was no decrease in thymic involution or increase in $\mathrm{T}$ cell output. Together, these data indicate that at least during aging, systemic IL-7 seems to influence thymic involution more directly than within the local thymic circuitry.

\section{GH and IGF-1}

We have performed a series of studies regarding the direct and indirect effects of GH on thymocytes and thymic microenvironment [for a review, see 40]. GH enhances thymic function through the activation of its receptors in TEC and thymocytes, a mechanism mediated by IGF-1. Exogenous GH stimulates proliferation of both cell types and an enhancement in the secretion of thymic hormones, cytokines, and chemokines by the thymic microenvironment. This leads to increased T-cell intrathymic trafficking and export, possibly by increasing the production of extracellular matrix and chemokines [41].

In addition to the endocrine role of $\mathrm{GH}$ in the thymus, the organ itself is able to produce and secrete $\mathrm{GH}$ by both developing thymocytes and TEC [40]. In fact, thymic GH contents diminish with advancing age, similar to systemic GH levels [42]. Accordingly, it is possible that thymusderived $\mathrm{GH}$ is also required for thymic maintenance.

\section{Luteinizing Hormone-Releasing Hormone}

We have previously demonstrated the use of luteinizing hormone-releasing hormone agonists (LHRH-A) for the enhancement of $\mathrm{T}$-cell recovery following autologous and allogeneic BM transplantation $[6,43]$. The chronic administration of LHRH-A downregulates the expression of its own receptor by the pituitary, which in turn downregulates the production of $\mathrm{LH}$ and FSH, leading to a blockade of sex hormone release. This has been demonstrated to result in beneficial effects on the BM and thymus, with increased thymopoiesis and naive T-cell production [6]. The role of locally produced LHRH in thymussenescenceis not yet elucidated; however, intrathymic production of LHRH increases in castrated mice, whereas testosterone replacement prevents this effect [44]. 
Fig. 2. Possible mechanisms by which therapies based on the stimulation of FoxN1 and activation of thymic epithelial precursor cells would be able to induce a sustained recovery of the aging thymus. a The therapies currently applied in clinics to enhance T-cell reconstitution are based on the exogenous administration of LHRH, IL-7, or $\mathrm{GH}$, but they result in a transient rejuvenation of thymopoiesis. b Therapies manipulating the expression of FoxN1 in the aging thymus induce improved TEC recovery and thymopoiesis. FoxN1 overexpression induces TEC proliferation (mainly TEC ${ }^{\text {hi }}$ ) and inhibits EMT, which results in adipocyte (A) formation from fibroblasts (F) converted from TECs (T). It has been suggested that FoxN1 has a central role in the induction of functionality and survival of a putative TEPC population. c A third possibility for cell-based therapy derives from the in vitro generation of TEPCs from hESCs or by reprogramming fibroblasts by forced FoxN1 expression. Further, the recent characterization of TEPCs in the adult thymus [47] with self-renewal and colonyforming potential, characterized as TEC ${ }^{\text {lo }}$, demonstrated that once activated, this subset could generate CTEC $^{\text {hi }}$ and mTEC $^{\text {lo }}$, and indirectly $\mathrm{mTEC}^{\text {hi }}$ from $\mathrm{mTEC}^{\mathrm{lo}}$. This potentially enables a novel strategy for therapeutic thymic regeneration by targeting endogenous TEPCs.

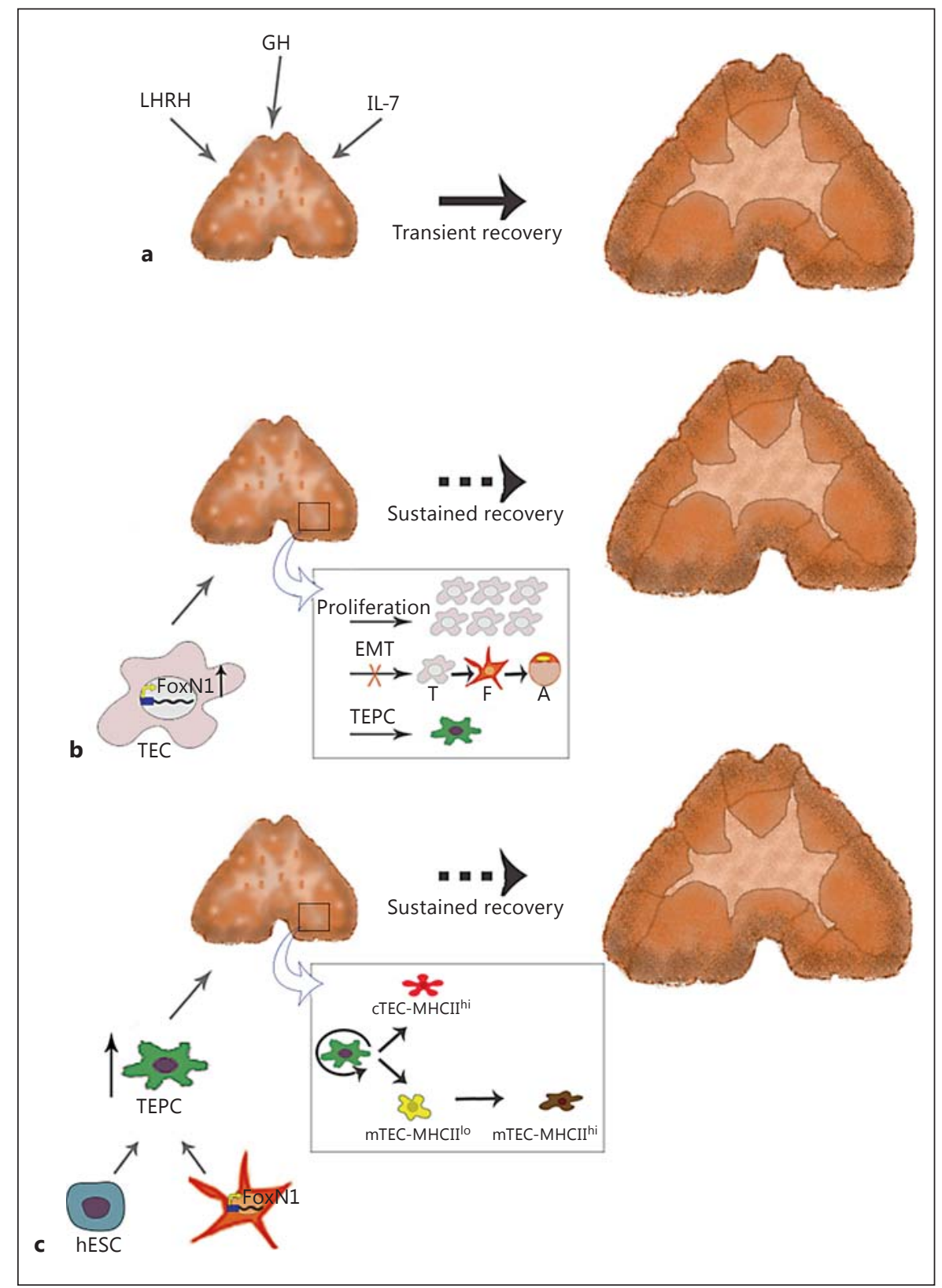

\section{Approaches for Thymic Regeneration}

The therapies currently applied in preclinical and clinical studies to enhance $\mathrm{T}$-cell reconstitution are based on the exogenous administration of LHRH-A, IL-7, IL-22, KGF and GH [for a review, see [45]. However, these treatments rely on substantial preexisting thymic tissue to support increased thymopoiesis, and the nature of TEC regeneration with LHRH-A may be transient (fig. 2a) [46]. In situations of significant thymic tissue damage, as may be the case with graft versus host disease or following multiple cytoablative treatments, it may be necessary to develop new approaches that focus on TEC regeneration for sustained thymic recovery (fig. 2). In this way, strategies to manipulate the expression of the critical TEC transcription factor forkhead box n1 (FoxN1) or the activation of TEPCs, recently identified in the adult thymus [47], provide novel strategies to potentially generate more sustained thymus rejuvenation in aging individuals. In addition, protocols have been developed for the directed 
Table 2. Efforts to rejuvenate the thymus through the manipulation of FoxN1 and generation of TEPCs from pluripotent stem cells

\begin{tabular}{|c|c|c|}
\hline Authors & Methods & Results \\
\hline $\begin{array}{l}\text { FoxN1 increase } \\
\text { Zook et al. [56] }\end{array}$ & Foxn1 transgenic mice (Foxn1Tg) & $\begin{array}{l}\text { Attenuation of age-associated thymic involution } \\
\text { Prevention of the expansion of peripheral CD } 4 \text { memory T cells } \\
\text { Attenuation in the decline of CD } 4^{+} \text {and } C D 8^{+} \text {naive cells }\end{array}$ \\
\hline Garfin et al. [55] & $\begin{array}{l}\text { Inactivation of } \mathrm{Rb} \text { family genes in } \\
\text { young mice }\end{array}$ & $\begin{array}{l}\text { Increased FoxN1 expression } \\
\text { Prevention of thymic involution during aging } \\
\text { Increased production of naive } \mathrm{T} \text { cells }\end{array}$ \\
\hline $\begin{array}{l}\text { Bredenkamp et al. } \\
\text { [57] }\end{array}$ & $\begin{array}{l}\text { TEC-specific increase of FoxN1 in the } \\
\text { thymus of aging mice }\end{array}$ & $\begin{array}{l}\text { Increased thymopoiesis } \\
\text { Increased naive T-cell output } \\
\text { Increasing proliferation of progenitor TEC }\left(\mathrm{MHCII}^{\mathrm{lo}}\right)\end{array}$ \\
\hline $\begin{array}{l}\text { TEPC generation } \\
\text { Lai and Jin [51] }\end{array}$ & Generation of TEPC from mESC & $\begin{array}{l}\text { Reconstitution of the normal thymic architecture in vivo } \\
\text { Thymocyte regeneration after BM transplantation } \\
\text { Increased number of functional naive splenic T cells }\end{array}$ \\
\hline Parent et al. [49] & Generation of TEPC from hESC & $\begin{array}{l}\text { T cell development upon transplantation into nude mice } \\
\text { Developed T cells capable of in vitro proliferation as well as in vivo } \\
\text { immune responses }\end{array}$ \\
\hline Sun et al. [50] & $\begin{array}{l}\text { hESC-derived TEPC transplantion in } \\
\text { NOD/SCID mice engrafted with HSCs }\end{array}$ & $\begin{array}{l}\text { In vivo differentiation into TEC expressing MHCII, AIRE, and FoxN1 } \\
\text { Human } T \text { cell generation in NOD/SCID mice engrafted with human HSCs }\end{array}$ \\
\hline
\end{tabular}

differentiation of pluripotent stem cells into TEPC in mice and humans [48-51], for the potential transplantation of de novo generated TEPC. These findings are summarized in table 2 and reviewed below.

\section{Manipulation of FoxN1 Expression}

FoxN1 is a transcription factor expressed in the thymus and skin epithelium. In the thymus, it is expressed exclusively by TECs and essential for thymic development [52]. Mutations in the FoxN1 gene in both humans and mice result in an athymic condition leading to severe immune deficiency. In affected patients with FoxN1 deficiency, the thymic lobes are still present, but thymopoiesis is completely blocked, leading to severe primary Tcell immunodeficiency and often death in early childhood due to severe infections [53]. Studies using a conditionally reversible null allele of Foxn 1 revealed that the expression of FoxN1 is required to differentiate the whole TEC network from TEPC and for the successful colonization of thymic rudiment by BM-derived precursors [54].

In addition to its role in organogenesis, FoxN1 coordinates TEC proliferation in both embryo and adult thymus through the regulation of S-phase genes. In mice mutant for the tumor suppressor retinoblastoma protein, the increased activity of E2F transcription factors resulted in the increased expression of FoxN1 and consequent proliferation of TEC subsets, while the downmodulation of FoxN1 in this mutant inhibited thymic expansion (fig. 2b) [55]. Accordingly, FoxN1 overexpression in aging mice induces a higher proliferation of MHCII ${ }^{\text {hi }}$ TECs and prevents structural alterations associated with thymic involution [56]. Recently, Bredenkamp et al. [57] demonstrated that the single regeneration of FoxN1 during aging results in robust thymic recovery, characterized by an attenuated decline in early thymic progenitors and thymocyte numbers and paralleled by a higher number of recent thymic emigrants in the periphery. 
FoxN1 impairment in thymic aging also seems to be related to the induction of TEC EMT and proadipose differentiation (fig. 2b). Accordingly, a prolonged decrease in FoxN1 and its regulator Wnt-4 leads to the degeneration of the thymic epithelial network [58], whereas Wnt-4 overexpression protects TECs against dexamethasone-induced senescence [59].

Consistent with the central role of FoxN1 in TEC homeostasis in the adult thymus through the induction of functionality and survival of TEPCs [60-62], Bredenkamp et al. [63] recently demonstrated that enforced FoxN1 expression alone was sufficient to reprogram mouse embryonic fibroblasts into functional TECs (iTECs). These cells were able to convert ETPs down the thymocyte lineage with $\mathrm{CD} 4^{+} \mathrm{CD} 8^{+}$double-positive cells and $\mathrm{CD}^{+}$and $\mathrm{CD} 8^{+}$single-positive cells when cocultured in vitro. When reaggregated with embryonic thymic mesenchyme and immature mouse thymocytes and grafted under the kidney capsule of nude mice, the iTECs were able to differentiate into all TEC subsets including Aire $^{+}$cells and form correctly compartmentalized corti$\mathrm{cal}$ and medullary regions. If this approach could directly translate to human cells using accessible patient tissue, it would potentially provide an autologous source of TECs for patients to be used either for direct transplantation or for in vitro generated autologous T cells (fig. 2c).

\section{Activation of TEPC for Thymic Regeneration}

The phenotypic identification and characterization of endogenous TEPCs in the thymus of adult mice has only recently been achieved [47], allowing a new field of exploration for the development of therapies targeting thymus rejuvenation.

Stem and progenitor cell populations are characterized by self-renewal and the capacity to give rise to different cell lineages. In epithelial tissue, homeostasis is maintained by epithelial stem cells via asymmetric division, enabling the repair of damaged tissues and self-renewal [64]. This process enables the progenitor cells to generate two different kinds of daughter cells: one continues to maintain multipotent potential, whereas the other differentiates into specialized cells responsible for the function of the tissue or organ.

TEC stem/progenitor cells have been identified in the early embryonic thymus (E12.5-E14.5) and are able to differentiate into the full range of thymic epithelial subsets, including Aire expressing mTEC [65-67]. Whether a common bipotent stem cell population exists in the postnatal thymus, which can form both cortical and medullary compartments to maintain homeostasis, has been

Improvement of the Thymic

Microenvironment through TECs a controversial topic for some time; however, an emerging body of data supports their existence.

Recently, an adult thymic epithelial progenitor population within the $\mathrm{TEC}^{\text {lo }}$ subset has been identified and characterized as bearing the phenotype $\mathrm{EpCAM}^{+} \mathrm{UEA}$ -

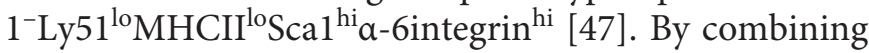
cellular and molecular characteristics, in vivo turnover and functional analysis by $3 \mathrm{D}$ in vivo and in vitro culture systems, their self-renewal was demonstrated as well as their colony forming potential and generation of mature cortical and medullary cell lineages, including the Aire ${ }^{+}$ medullary subset. Whether FoxN1 expression is absolutely required in adult thymic epithelial stem cells has recently been challenged in an article by Ucar et al. [68]; however, this will no doubt continue to be debated.

By means of a transgenic label-retaining cell assay, another recent study revealed in neonatal and adult mice the existence of nonsenescent, quiescent cTECs with putative features of progenitor cells, expressing relatively low levels of p16INK4a, p19ARF, and Serpine1 and high levels of Bmi1, Foxn1, Trp63, and Wnt4 [69]. These cells were only detectable after 16 weeks in adults, but not in neonate or young mice. Interestingly, it was demonstrated that the dramatic increase of the mTEC/cTEC ratio during the first weeks of life occurs independent of apoptosis or proliferation modulation, favoring the hypothesis of generation of mTEC phenotype from cTEC [69].

In summary, the identification of adult TEPC opens a new field to progress research into the molecular regulation of these cells during homeostasis, differentiation and age-dependent degeneration, besides their manipulation for regeneration following damage.

\section{TEPC Generation from Pluripotent Stem Cells}

Pluripotent stem cells hold great promise in the field of regenerative medicine. The ability to selectively induce the generation of TEPCs in vitro from ESCs or induced pluripotent stem cells for transplantation has important implications regarding the prevention and/or treatment of primary and secondary T-cell immunodeficiencies [50, 70].

The generation of TEPC from pluripotent stem cells was first demonstrated using mouse ESCs (mESCs), which were selectively induced in vitro to differentiate into cells that have the phenotype of TEPCs [51]. In this elegant study, Lai and Jin [50] demonstrated that, when placed in vivo, these mESC-derived TEPCs self-renew, develop into TECs, and reconstitute the normal thymic architecture. Functionally, these mESC-derived TEPCs enhanced thymocyte regeneration after BM transplanta- 
tion and increased the number of functional naive splenic T cells. Later, Lai et al. [70] showed that transplantation of mESC-derived TEPCs resulted in the efficient generation of naive $\mathrm{T}$ cells in both young and old recipients following allogeneic BM transplantation.

The development of protocols for human ESCs (hESCs) to generate TECs capable of supporting T-cell development, followed a more complex process of mimicking thymus organogenesis $[49,50]$. Sun et al. [50] directed the differentiation of hESCs into TEPCs by sequential regulation of Activin, retinoic acid, BMP, and WNT signals to initially differentiate cells into definitive endoderm and then generate TEPC identified by the expression of FoxN1 [49]. Cells underwent final development in vivo, with the induced thymic epithelium expressing the functional thymic markers MHCII and AIRE. These human TECs could support mouse thymopoiesis in T-cell-deficient mice and promote human Tcell generation in nonobese diabetic/severe combined immunodeficiency mice. The study by Parent et al. [50] included a more precise regulation of TGF- $\beta$, BMP4, retinoic acid, Wnt, Shh, and FGF, signaling the maturation of hESC-derived TEPC into functional TECs that supported T-cell development [49]. Importantly, the engrafted TEPCs produced $\mathrm{T}$ cells capable of in vitro proliferation as well as in vivo immune responses. A third study using a FoxN1-GFP/w hESC reporter line cultured hESC in high levels of Activin A to form definitive endoderm followed by KGF under serum-free conditions [48]. The generation of FoxN1 ${ }^{+}\left(\mathrm{GFP}^{+}\right)$thymic endodermal progenitors was achieved through the sequential expression of genes involved in endoderm and thymus development. Through the transcriptional profiling of purified FoxN1$\mathrm{GFP}^{+}$cells, Soh et al. [48] identified several combinations of cell-surface markers as ITGB4, HLA-DR, and EpCAM that could selectively isolate FoxN1 ${ }^{+}$TEC progenitor populations derived from unmodified ESC lines. This protocol was also demonstrated to be reproducible using alternative hESC lines and an induced pluripotent stem cell line. Thus, pluripotent stem cell-derived TEPC grafts, using the patient's own cells, may lead to broad applications for restoring immunity following irreversible thymic damage, or age-related thymic decline (fig. 2c).

\section{Conclusion}

The rejuvenation of the immune system can be achieved by therapies focused on thymus reestablishment. Like in other epithelial tissues, the postnatal thy- mus appears to preserve an intrinsic ability to regenerate by responding to external stimuli, such as sex steroid ablation and cytokine administration. In particular, the recovery of the TEC compartment is a determinant for sustained restoration of thymopoiesis. Current approaches focus on the modulation of growth factors and hormones secreted by TECs, and therefore only promote a partial and transient thymus recovery. Thus, the emergence of new strategies based on TEC recovery, such as inducing FoxN1 expression, TEPC activation and de novo generation of TEPC, have emerged as a new generation of strategic interventions focused on TEC reestablishment.

The recent identification of progenitor markers and their consecutive expression pattern in the adult thymus is an important step in the analysis of the prospective potential of TEPCs as well as for establishing strategies to regenerate a functional thymus with full cortical and medullary compartments. Further, the actual results achieved with the development of TEPCs from pluripotent stem cells able to generate functional human $\mathrm{T}$ cells in vivo has pioneered a new field of clinical and therapeutic possibilities for the treatment of pathologies related to T cell-associated immunosuppression. Regarding the potential manipulation of FoxN1 for TEC generation, the identification of the precise pathways that modulate the expression of this transcription factor provide a new paradigm for regenerative biology strategies. The combination of strategies focusing on both TEC and lymphoid regeneration may achieve a therapeutic threshold that will successfully enhance full immune recovery following cytoablative treatments in the aged and elderly.

\section{Acknowledgements}

This work was supported by grants from CNPq, Capes, Faperj and Fiocruz (Brazil); FOCEM (Mercosur); National Health and Medical Research Council and the Victorian Government - California Institute for Regenerative Medicine (Australia).

References

$$
\begin{aligned}
& \text { Steinmann GG, Klaus B, Muller-Hermelink } \\
& \text { HK: The involution of the ageing human thy- } \\
& \text { mic epithelium is independent of puberty. A } \\
& \text { morphometric study. Scand J Immunol 1985; } \\
& \text { 22:563-575. } \\
& 2 \text { Qi Q, Zhang DW, Weyand CM, Goronzy JJ: } \\
& \text { Mechanisms shaping the naive T cell reper- } \\
& \text { toire in the elderly - thymic involution or pe- } \\
& \text { ripheral homeostatic proliferation? Exp } \\
& \text { Gerontol 2014;54:71-74. }
\end{aligned}
$$


-3 Mackall CL, Fleisher TA, Brown MR, Andrich MP, Chen CC, Feuerstein IM, et al: Age, thymopoiesis, and CD4+ T-lymphocyte regeneration after intensive chemotherapy. $\mathrm{N}$ Engl J Med 1995;332:143-149.

-4 Dudakov JA, Khong DM, Boyd RL, Chidgey AP: Feeding the fire: the role of defective bone marrow function in exacerbating thymic involution. Trends Immunol 2010;31:191-198.

-5 Goldberg GL, Zakrzewski JL, Perales MA, van den Brink MR: Clinical strategies to enhance T cell reconstitution. Semin Immunol 2007; 19:289-296.

6 Goldberg GL, King CG, Nejat RA, Suh DY, Smith OM, Bretz JC, et al: Luteinizing hormone-releasing hormone enhances $\mathrm{T}$ cell recovery following allogeneic bone marrow transplantation. J Immunol 2009;182:58465854.

7 Sauce D, Larsen M, Fastenackels S, Duperrier A, Keller M, Grubeck-Loebenstein B, et al: Evidence of premature immune aging in patients thymectomized during early childhood. J Clin Invest 2009;119:3070-3078.

8 Appay V, Sauce D, Prelog M: The role of the thymus in immunosenescence: lessons from the study of thymectomized individuals. Aging 2010;2:78-81.

-9 van Gent R, Schadenberg AW, Otto SA, Nievelstein RA, Sieswerda GT, Haas F, et al: Longterm restoration of the human T-cell compartment after thymectomy during infancy: a role for thymic regeneration? Blood 2011;118: 627-634.

10 Ventevogel MS, Sempowski GD: Thymic rejuvenation and aging. Curr Opin Immunol 2013;25:516-522.

11 Dixit VD: Thymic fatness and approaches to enhance thymopoietic fitness in aging. Curr Opin Immunol 2010;22:521-528.

12 Gray DH, Seach N, Ueno T, Milton MK, Liston A, Lew AM, et al: Developmental kinetics, turnover, and stimulatory capacity of thymic epithelial cells. Blood 2006;108:3777-3785.

13 Zhu M, Fu Y: The complicated role of NF- $\mathrm{kB}$ in T-cell selection. Cell Mol Immunol 2010;7: 89-93.

14 Klug DB, Crouch E, Carter C, Coghlan L, Conti CJ, Richie ER: Transgenic expression of cyclin D1 in thymic epithelial precursors promotes epithelial and T cell development. J Immunol 2000;164:1881-1888.

15 Jenkinson WE, Bacon A, White AJ, Anderson G, Jenkinson EJ: An epithelial progenitor pool regulates thymus growth. J Immunol 2008; 181:6101-6108.

16 Mackall CL, Punt JA, Morgan P, Farr AG, Gress RE: Thymic function in young/old chimeras: substantial thymic $\mathrm{T}$ cell regenerative capacity despite irreversible age-associated thymic involution. Eur J Immunol 1998;28: 1886-1893.

17 Yang H, Youm YH, Dixit VD: Inhibition of thymic adipogenesis by caloric restriction is coupled with reduction in age-related thymic involution. J Immunol 2009; 183:3040-3052.
8 Youm YH, Yang H, Sun Y, Smith RG, Manley NR, Vandanmagsar B, et al: Deficient ghrelin receptor-mediated signaling compromises thymic stromal cell microenvironment by accelerating thymic adiposity. J Biol Chem 2009;284:7068-7077.

19 Mohtashami M, Shah DK, Nakase H, Kianizad K, Petrie HT, Zuniga-Pflucker JC: Direct comparison of Dll1- and Dll4-mediated Notch activation levels shows differential lymphomyeloid lineage commitment outcomes. J Immunol 2010;185:867-876.

20 Min H, Montecino-Rodriguez E, Dorshkind $\mathrm{K}$ : Reduction in the developmental potential of intrathymic T cell progenitors with age. J Immunol 2004;173:245-250.

-21 Heng TS, Goldberg GL, Gray DH, Sutherland JS, Chidgey AP, Boyd RL: Effects of castration on thymocyte development in two different models of thymic involution. J Immunol 2005; 175:2982-2993.

22 Lagresle C, Gardie B, Eyquem S, Fasseu M, Vieville JC, Pla M, et al: Transgenic expression of the p16(INK4a) cyclin-dependent kinase inhibitor leads to enhanced apoptosis and differentiation arrest of CD4-CD8- immature thymocytes. J Immunol 2002;168: 2325-2331.

23 Gui J, Zhu X, Dohkan J, Cheng L, Barnes PF, Su DM: The aged thymus shows normal recruitment of lymphohematopoietic progenitors but has defects in thymic epithelial cells. Int Immunol 2007;19:1201-1211.

24 Aspinall R: Age-associated thymic atrophy in the mouse is due to a deficiency affecting rearrangement of the TCR during intrathymic $\mathrm{T}$ cell development. J Immunol 1997;158:30373045.

25 Lee JB, Oelke M, Ramachandra L, Canaday DH, Schneck JP: Decline of influenza-specific CD8+ T cell repertoire in healthy geriatric donors. Immun Ageing 2011;8:6.

26 Savino W, Dardenne M: Neuroendocrine control of thymus physiology. Endocr Rev 2000;21:412-443.

27 Yarilin AA, Belyakov IM: Cytokines in the thymus: production and biological effects. Curr Med Chem 2004;11:447-464.

28 Lepletier A, de Carvalho VF, Rodrigues e Silva PM, Villar S, Perez AR, Savino W, et al: Trypanosoma cruzi disrupts thymic homeostasis by altering intrathymic and systemic stressrelated endocrine circuitries. PLoS Negl Trop Dis 2013;7:e2470.

29 Sempowski GD, Hale LP, Sundy JS, Massey JM, Koup RA, Douek DC, et al: Leukemia inhibitory factor, oncostatin M, IL-6, and stem cell factor mRNA expression in human thymus increases with age and is associated with thymic atrophy. J Immunol 2000;164:21802187.

30 Ellingsworth LR, Nakayama D, Segarini P, Dasch J, Carrillo P, Waegell W: Transforming growth factor-beta $s$ are equipotent growth inhibitors of interleukin-1-induced thymocyte proliferation. Cell Immunol 1988;114: $41-54$.
Takahama Y, Letterio JJ, Suzuki H, Farr AG, Singer A: Early progression of thymocytes along the $\mathrm{CD} 4 / \mathrm{CD} 8$ developmental pathway is regulated by a subset of thymic epithelial cells expressing transforming growth factor beta. J Exp Med 1994;179:1495-1506.

32 Hauri-Hohl MM, Zuklys S, Keller MP, Jeker LT, Barthlott T, Moon AM, et al: TGF-beta signaling in thymic epithelial cells regulates thymic involution and postirradiation reconstitution. Blood 2008;112:626-634.

-33 Jeker LT, Barthlott T, Keller MP, Zuklys S, Hauri-Hohl M, Deng CX, et al: Maintenance of a normal thymic microenvironment and $\mathrm{T}$ cell homeostasis require Smad4-mediated signaling in thymic epithelial cells. Blood 2008;112:3688-3695.

34 Leask A, Abraham DJ: TGF-beta signaling and the fibrotic response. FASEB J 2004;18: 816-827.

-35 Oosterwegel MA, Haks MC, Jeffry U, Murray R, Kruisbeek AM: Induction of TCR gene rearrangements in uncommitted stem cells by a subset of IL-7 producing, MHC class-II-expressing thymic stromal cells. Immunity 1997;6:351-360.

-36 von Freeden-Jeffry U, Vieira P, Lucian LA, McNeil T, Burdach SE, Murray R: Lymphopenia in interleukin (IL)-7 gene-deleted mice identifies IL-7 as a nonredundant cytokine. J Exp Med 1995;181:1519-1526.

37 Andrew D, Aspinall R: Age-associated thymic atrophy is linked to a decline in IL-7 production. Exp Gerontol 2002;37:455-463.

38 Bhatia SK, Tygrett LT, Grabstein KH, Waldschmidt TJ: The effect of in vivo IL-7 deprivation on T cell maturation. J Exp Med 1995; 181:1399-1409.

-39 Phillips JA, Brondstetter TI, English CA, Lee HE, Virts EL, Thoman ML: IL-7 gene therapy in aging restores early thymopoiesis without reversing involution. J Immunol 2004;173: 4867-4874.

40 Savino W, Dardenne M: Pleiotropic modulation of thymic functions by growth hormone: from physiology to therapy. Curr Opin Pharmacol 2010;10:434-442.

-41 Smaniotto S, de Mello-Coelho V, Villa-Verde DM, Pleau JM, Postel-Vinay MC, Dardenne $\mathrm{M}$, et al: Growth hormone modulates thymocyte development in vivo through a combined action of laminin and CXC chemokine ligand 12. Endocrinology 2005;146:3005-3017.

42 Taub DD, Murphy WJ, Longo DL: Rejuvenation of the aging thymus: growth hormonemediated and ghrelin-mediated signaling pathways. Curr Opin Pharmacol 2010;10: 408-424

43 Sutherland JS, Spyroglou L, Muirhead JL, Heng TS, Prieto-Hinojosa A, Prince HM, et al: Enhanced immune system regeneration in humans following allogeneic or autologous hemopoietic stem cell transplantation by temporary sex steroid blockade. Clin Cancer Res 2008;14:1138-1149.
Improvement of the Thymic

Microenvironment through TECs
Gerontology 2015;61:504-514 DOI: $10.1159 / 000375160$ 
-44 Azad N, LaPaglia N, Agrawal L, Steiner J, Uddin S, Williams DW, et al: The role of gonadectomy and testosterone replacement on thymic luteinizing hormone-releasing hormone production. J Endocrinol 1998;158:229-235.

-45 Velardi E, Dudakov JA, van den Brink MR: Clinical strategies to enhance thymic recovery after allogeneic hematopoietic stem cell transplantation. Immunol Lett 2013;155:31-35.

46 Griffith AV, Fallahi M, Venables T, Petrie HT: Persistent degenerative changes in thymic organ function revealed by an inducible model of organ regrowth. Aging Cell 2012;11:169177.

47 Wong K, Liser NL, Barsanti M, Lim JM, Hammet MV, Khong DM, Siatskas C, Gray DH, Boyd RL, Chidgey AP: Multilineage potential and self-renewal define an epithelial progenitor cell population in the adult thymus. Cell Rep 2014;8:1198-1209.

-48 Soh CL, Giudice A, Jenny RA, Elliott DA, Hatzistavrou T, Micallef SJ, et al: FOXN1 $1^{\mathrm{GFP} / \mathrm{w}}$ reporter hESCs enable identification of integrin- $\beta 4$, HLA-DR, and EpCAM as markers of human PSC-derived $\mathrm{FOXN1}{ }^{+}$ thymic epithelial progenitors. Stem Cell Reports 2014;2:925-937.

49 Parent AV, Russ HA, Khan IS, LaFlam TN, Metzger TC, Anderson MS, Hebrok M: Generation of functional thymic epithelium from human embryonic stem cells that supports host T cell development. Cell Stem Cell 2013; 13:219-229.

-50 Sun X, Xu J, Lu H, Liu W, Miao Z, Sui X, et al: Directed differentiation of human embryonic stem cells into thymic epithelial progenitorlike cells reconstitutes the thymic microenvironment in vivo. Cell Stem Cell 2013;13:230236.

51 Lai L, Jin J: Generation of thymic epithelial cell progenitors by mouse embryonic stem cells. Stem Cells 2009;27:3012-3020.

52 Nehls M, Pfeifer D, Schorpp M, Hedrich H, Boehm T: New member of the winged-helix protein family disrupted in mouse and rat nude mutations. Nature 1994;372:103-107.

53 Frank J, Pignata C, Panteleyev AA, Prowse DM, Baden H, Weiner L, et al: Exposing the human nude phenotype. Nature 1999;398: 473-474.
54 Nowell CS, Bredenkamp N, Tetelin S, Jin X, Tischner C, Vaidya H, et al: Foxn1 regulates lineage progression in cortical and medullary thymic epithelial cells but is dispensable for medullary sublineage divergence. PLoS Genet 2011;7:e1002348.

55 Garfin PM, Min D, Bryson JL, Serwold T, Edris B, Blackburn CC, et al: Inactivation of the RB family prevents thymus involution and promotes thymic function by direct control of Foxn1 expression. J Exp Med 2013;210: 1087-1097.

56 Zook EC, Krishack PA, Zhang S, Zeleznik-Le NJ, Firulli AB, Witte PL, et al: Overexpression of Foxn 1 attenuates age-associated thymic involution and prevents the expansion of peripheral CD4 memory T cells. Blood 2011; 118:5723-5731.

57 Bredenkamp N, Nowell CS, Blackburn CC: Regeneration of the aged thymus by a single transcription factor. Development 2014;141: 1627-1637.

58 Kvell K, Varecza Z, Bartis D, Hesse S, Parnell S, Anderson G, et al: Wnt4 and LAP2alpha as pacemakers of thymic epithelial senescence. PLoS One 2010;5:e10701.

-59 Talaber G, Kvell K, Varecza Z, Boldizsar F, Parnell SM, Jenkinson EJ, et al: Wnt-4 protects thymic epithelial cells against dexamethasone-induced senescence. Rejuvenation Res 2011;14:241-248.

60 Corbeaux T, Hess I, Swann JB, Kanzler B, Haas-Assenbaum A, Boehm T: Thymopoiesis in mice depends on a Foxn1-positive thymic epithelial cell lineage. Proc Natl Acad Sci USA 2010;107:16613-16638.

61 Bleul CC, Corbeaux T, Reuter A, Fisch P, Monting JS, Boehm T: Formation of a functional thymus initiated by a postnatal epithelial progenitor cell. Nature 2006;441:992-996.

62 Burnley P, Rahman M, Wang H, Zhang Z, Sun X, Zhuge Q, et al: Role of the p63-FoxN1 regulatory axis in thymic epithelial cell homeostasis during aging. Cell Death Dis 2013; 4:e932.
63 Bredenkamp N, Ulyanchenko S, O’Neill KE, Manley NR, Vaidya HJ, Blackburn CC: An organized and functional thymus generated from FOXN1-reprogrammed fibroblasts. Nat Cell Biol 2014;16:902-908.

-64 Blanpain C, Horsley V, Fuchs E: Epithelial stem cells: turning over new leaves. Cell 2007; 128:445-458

65 Rossi SW, Jenkinson WE, Anderson G, Jenkinson EJ: Clonal analysis reveals a common progenitor for thymic cortical and medullary epithelium. Nature 2006;441:988-991.

66 Baik S, Jenkinson EJ, Lane PJ, Anderson G, Jenkinson WE: Generation of both cortical and Aire ${ }^{+}$medullary thymic epithelial compartments from $\mathrm{CD} 205^{+}$progenitors. Eur J Immunol 2013;43:589-594.

67 Gill J, Malin M, Hollander GA, Boyd R: Generation of a complete thymic microenvironment by MTS $24^{+}$thymic epithelial cells. Nat Immunol 2002;3:635-642.

- 68 Ucar A, Ucar O, Klug P, Matt S, Brunk F, Hofmann TG, et al: Adult thymus contains FoxN1- epithelial stem cells that are bipotent for medullary and cortical thymic epithelial lineages. Immunity 2014;41:257-269.

69 Dumont-Lagacé M, Brochu S, St-Pierre C, Perreault C: Adult thymic epithelium contains nonsenescent label-retaining cells. J Immunol 2014;192:2219-2226.

-70 Lai L, Cui C, Jin J, Hao Z, Zheng Q, Ying M, et al: Mouse embryonic stem cell-derived thymic epithelial cell progenitors enhance T-cell reconstitution after allogeneic bone marrow transplantation. Blood 2011;118:3410-3418.

-71 Haynes BF, Sempowski GD, Wells AF, Hale LP: The human thymus during aging. Immunol Res 2000;22:253-261.

-72 Mello Coelho V, Bunbury A, Rangel LB, Giri B, Weeraratna A, Morin PJ, et al: Fat-storing multilocular cells expressing CCR5 increase in the thymus with advancing age: potential role for CCR5 ligands on the differentiation and migration of preadipocytes. Int J Med Sci 2009;7:1-14.

-73 Coin Aragüez L, Murri M, Oliva Olivera W, Salas J, Mayas MD, Delgado-Lista J, et al: Thymus fat as an attractive source of angiogenic factors in elderly subjects with myocardial ischemia. Age (Dordr) 2013;35:1263-1275. 